\title{
A comparative analysis of metal allergens associated with dental alloy prostheses and the expression of HLA-DR in gingival tissue
}

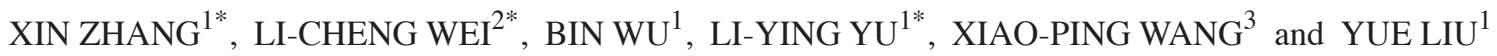 \\ ${ }^{1}$ Department of Stomatology, Huashan Hospital, Fudan University, Shanghai 200040; ${ }^{2}$ Department of Orthopaedics, \\ Shanghai Guanghua Hospital of Integrated Traditional Chinese and Western Medicine, Shanghai 200052; \\ ${ }^{3}$ Department of Stomatology, Yuhuangding Hospital, Yantai, Shandong 264000, P.R. China
}

Received December 26, 2014; Accepted September 22, 2015

DOI: $10.3892 / \mathrm{mmr} .2015 .4562$

\begin{abstract}
The present study aimed to provide guidance for the selection of prosthodontic materials and the management of patients with a suspected metal allergy. This included a comparison of the sensitivity of patients to alloys used in prescribed metal-containing prostheses, and correlation analysis between metal allergy and accompanying clinical symptoms of sensitized patients using a patch test. The results from the patch test and metal component analyses were processed to reach a final diagnosis. In the present study, four dental alloys were assessed. Subsequent to polishing the surface of a metal restoration, the components were analyzed using an X-ray fluorescence microscopy and spectrometry. Immunohistochemical analysis, reverse transcription-polymerase chain reaction and western blotting were used to detect the expression levels of human leukocyte antigen (HLA)-DR in gingival tissues affected by alloy restoration, and in normal gingival tissue samples. Positive allergens identified in the patch test were consistent with the components of the metal prostheses. The prevalence of nickel (Ni) allergy was highest $(22.8 \%)$, and women were significantly more allergic to palladium and $\mathrm{Ni}$ than men $(\mathrm{P}<0.05)$. The protein and gene expression levels of HLA-DR in the Ni-chromium $(\mathrm{Cr})$ prosthesis group were significantly higher, compared with those in the other groups $(\mathrm{P}<0.01)$; followed by cobalt-Cr alloy, gold alloy and titanium alloy. In conclusion, dentists require an understanding of the corrosion and allergy rates of prescribed alloys, in order to reduce the risk of allergic reactions. Patch testing for hypersensitive patients
\end{abstract}

Correspondence to: Professor Yue Liu, Department of Stomatology, Huashan Hospital, Fudan University, 12 Middle Urumqi Road, Shanghai 200040, P.R. China

E-mail: liuyue20131124@163.com

*Contributed equally

Key words: metal allergy, patch test, allergic reactions, human leukocyte antigen-DR, X-ray fluorescence microscope spectrometer is recommended and caution is required when planning to use different alloys in the mouth.

\section{Introduction}

In dentistry, $>20$ metallic elements are processed into various types of dental metal alloy. These alloys are then cast and processed for use as metal restorations. Previous studies have demonstrated that various symptoms are associated with different metals $(1,2)$. Nickel $(\mathrm{Ni})$, chromium $(\mathrm{Cr})$, mercury $(\mathrm{Hg})$, palladium $(\mathrm{Pd})$ and cobalt $(\mathrm{Co})$ are metals, which are commonly used in dentistry, and have been known to cause allergies. Allergic reactions to these materials occur not only in the mucosa of the oral cavity, but also on the hands, feet and entire body $(3,4)$. A previous study performed a patch test on 212 patients with suspicious metal allergies, and demonstrated that $\mathrm{Ni}$ exhibited the highest rate of positivity (25\%), followed by Pd (24.4\%), Cr (16.7\%) and Co (15.9\%) (5). Patch tests are considered the most reliable method for the diagnosis of a metal allergy. When the allergic antigen is a metal ion, primary irritation responses occur readily, and it is often difficult to distinguish irritation from allergic reactions. In the case of type IV hypersensitivity, patch tests are usually used to determine the allergen, and are considered the gold standard in the diagnosis of type IV hypersensitivity reactions (6). Studies have shown that the levels of HLA-DR expression allows the identification of patients with clinical marginal rejection.

Metals used in dentistry can lead to metal sensitization, and the sensitization rates of metals differ (7). The present study used a patch test to comparatively analyze the various metal allergic reactions of patients, who had undergone repair work or dental restorations using oral metals, in order to provide guidance for dentists in terms of the selection of appropriate metals, as well as to provide a reference for patients with oral mucosa and skin diseases. In addition, the present study aimed to identify the most suitable metallic material in order to provide a foundation for patient treatment.

\section{Materials and methods}

Subjects. The present study was performed by recruitment of 92 outpatients of dental clinics in Huashan Hospital (Shanghai, 
China) between September 2011 and December 2012. The inclusion criteria were as follows: (i) All patients (age, 18-65 years; 43 male, 49 female) provided written, informed consent prior to involvement in the investigation, were able to receive tests in accordance with the program requirements and attend follow-up sessions; (ii) no lesions were present on the tested area; (iii) patients had previously received an alloy restoration in the oral cavity; (iv) patients had stopped using oral antihistamines at least 3 days, and systemic corticosteroids and immunosuppressive drugs at least 2 weeks prior to the start of the investigation; (v) topical systemic corticosteroids and immunosuppressive drugs had not been applied to the test site at least 2 weeks prior to the start of the investigation.

The present study was approved by the Ethics Committee of the Huashan Hospital affiliated to Fudan University (Shanghai, China).

Patch test evaluation method. Using the Ruimin patch series (Chemotechnique MB Diagnostics AB, Vellinge, Sweden), 20 allergens were assessed, including 19 types of metal allergens and a control (Vaseline ${ }^{\circledR}$; Unilever, London, UK). The metal allergens assessed comprised normal metal components contained in dental restorations (Table I). The patch test (positioned on the back skin on eithe side of the spine) was performed using an IQ Test Core Chamber (Nanjing Allergy Biotechnology Co.,. Ltd., Jiangsu, China). The International Contact Dermatitis Group's recommended patch test recording method was adopted, as follows: +++ , strong positive reaction (erythema, significant invasion, papula, blisters, bullous pemphigoid); ++ , positive reaction (erythema, invasion, papula, blisters); +, weak positive reaction (erythema, invasion, small pimple); ?+, suspicious reaction (mild erythema); -, negative reaction; IR, irritation; NT, not tested. +, ++ and +++ were considered a positive allergic reaction (8).

Metal component assessment. An X-ray fluorescence microscope spectrometer (XFMS; XGT-5000XIISL, HORIBA Trading Co., Ltd., Shanghai, Japan) was used to detect metal components. The measurements were performed using a charge-coupled device camera. This method is able to detect elements in the periodic table from ${ }^{11} \mathrm{Na}$ to ${ }^{92} \mathrm{U}$, with a resolution $\leq 150 \mathrm{eV}$, measurement range/accuracy of $0-40.96 \mathrm{keV}$, temperature of $23^{\circ} \mathrm{C}$ and relative humidity of $55 \%$.

Silicone OneGloss (Japanese Pine Corp., Kariya, Japan) was specifically developed for the repair, polishing and shaping of resin and glass ionomer. Silicon particles were removed, and XFMS was used to detect the metal components.

Immunohistochemical analysis. Gingival tissues were fixed in $4 \%$ paraformaldehyde (Sangon Biotech Co., Ltd., Shanghai, China) for $24 \mathrm{~h}$ and embedded in paraffin (Sangon Biotech Co., Ltd.). The tissue was cut into $4 \mathrm{~mm}$ sections, blocked in $0.5 \%$ bovine serum albumin (Sigma-Aldrich, St. Louis, MO, USA) for $30 \mathrm{~min}$ and incubated with rabbit anti-human leukocyte antigen (HLA)-DR polyclonal antibody (cat. no. ab175085; Abcam, Cambridge, UK; dilution 1:1,000) overnight at $4^{\circ} \mathrm{C}$ in a humid chamber, prior to being washed three times with $0.01 \mathrm{M}$ phosphate-buffered saline (PBS). The tissue samples were subsequently incubated with secondary antibody (goat antirabbit/mouse; Dako, Glostrup, Denmark) at $37^{\circ} \mathrm{C}$ for $1 \mathrm{~h}$, prior
Table I. Allergen composition and concentration in the patch test.

\begin{tabular}{rlr}
\hline & & \multicolumn{1}{c}{$\begin{array}{r}\text { Concentration } \\
\text { No. }\end{array}$} \\
\hline 1 & \multicolumn{1}{c}{ Allergen } \\
2 & $\mathrm{H}_{12} \mathrm{AlCl}_{3} \mathrm{O}_{6}$ & 2.0 \\
3 & $\mathrm{Na}_{3} \mathrm{Au}\left(\mathrm{S}_{2} \mathrm{O}_{3}\right)_{2} \cdot 2 \mathrm{H}_{2} \mathrm{O}$ & 2.0 \\
4 & $\mathrm{SnO}_{2}$ & 1.0 \\
5 & $\mathrm{FeCl}_{3}$ & 2.0 \\
6 & $\left(\mathrm{NH}_{4}\right)_{2} \mathrm{PtCl}_{6}$ & 0.1 \\
7 & $\mathrm{PdCl}_{2}$ & 2.0 \\
8 & $\mathrm{InCl}_{3}$ & 10.0 \\
9 & $\mathrm{IrCl}_{3}$ & 1.0 \\
10 & $\mathrm{ZnCl}_{2}$ & 1.0 \\
11 & $\mathrm{MnO}_{2}$ & 2.0 \\
12 & $\mathrm{AgNO}_{3}$ & 1.0 \\
13 & $\mathrm{Cr}_{2} \mathrm{~K}_{2} \mathrm{O}_{7}$ & 0.5 \\
14 & $\mathrm{CoCl}_{2}$ & 1.0 \\
15 & $\mathrm{CuSO}_{4}$ & 2.0 \\
16 & $\mathrm{HgCl}_{2}$ & 0.1 \\
17 & $\mathrm{NiSO}_{4}$ & 5.0 \\
18 & $\mathrm{CdCl}_{2}$ & 1.0 \\
19 & $\mathrm{H}_{8} \mathrm{MoN}_{2} \mathrm{O}_{4}$ & 1.0 \\
20 & $\mathrm{TiC}_{2} \mathrm{O}_{4}$ & 5.0 \\
& $\mathrm{Vaseline}^{\circledR}$ & 100.0 \\
\hline & &
\end{tabular}

to being washed three times with $0.01 \mathrm{M}$ PBS. The immune complex was visualized using a Dako REAL ${ }^{\mathrm{TM}}$ EnVision $^{\mathrm{TM}}$ Detection system containing peroxidase/DAB, according to the manufacturer's protocol. The nuclei were counterstained with hematoxylin (Sangon Biotech Co., Ltd.), and the sections were observed under a Nikon Eclipse 50i microscope (Nikon Corporation, Tokyo, Japan).

RNA isolation and reverse transcription-quantitative polymerase chain reaction $(R T-q P C R)$. The tissue samples $(100 \mathrm{mg})$ and TRIzol ${ }^{\circledR}$ reagent $(1 \mathrm{ml}$; Invitrogen; Thermo Fisher Scientific, Inc., Waltham, MA, USA) were homogenized in a homogenate machine at $120 \mathrm{hz}$ for $5 \mathrm{~min}$, and then centrifuged at $12,000 \mathrm{x}$ g for $15 \mathrm{~min}$ at $4^{\circ} \mathrm{C}$ in order to obtain the supernatant. Total RNA $(1 \mu \mathrm{g})$ was isolated from the gingival tissues $\left(3 \mathrm{~cm}^{3}\right)$ obtained from the patients who exhibited allergic reactions using TRIzol ${ }^{\circledR}$ reagent and was converted into cDNA using a cDNA synthesis kit (cat. no. DRR037A; Takara Bio, Inc., Otsu, Japan), according to the manufacturer's protocol. RT-qPCR was performed to determine the expression levels of HLA-DR using SYBR Supermix (Takara Bio Inc., Otsu, Japan) and RT-qPCR Supermix (Takara Bio, Inc.) with the following thermocycling conditions: Denaturation at $95^{\circ} \mathrm{C}$ for $30 \mathrm{sec}$; annealing at $95^{\circ} \mathrm{C}$ for $5 \mathrm{sec}$; and extension at $60^{\circ} \mathrm{C}$ for $30 \mathrm{sec}$ for 40 cycles. The relative expression levels of HLA-DR were calculated using the $2^{-\Delta(\Delta \mathrm{Cq})}$ method (9). The expression of the HLA-DR transcripts were normalized to the expression of glyceraldehyde 3-phosphate dehydrogenase (GAPDH) in the same sample. Primer sequences (Sangon Biotech Co., Ltd.) 


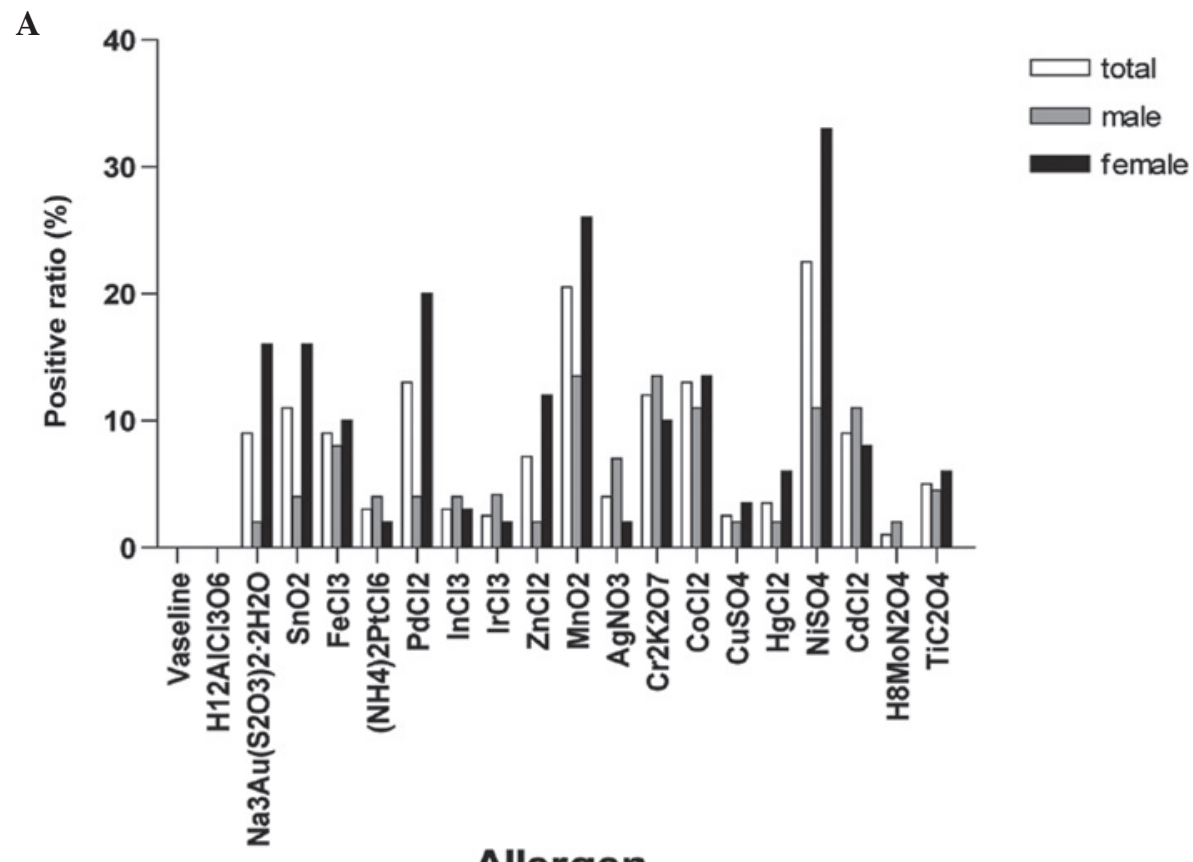

B

Allergen

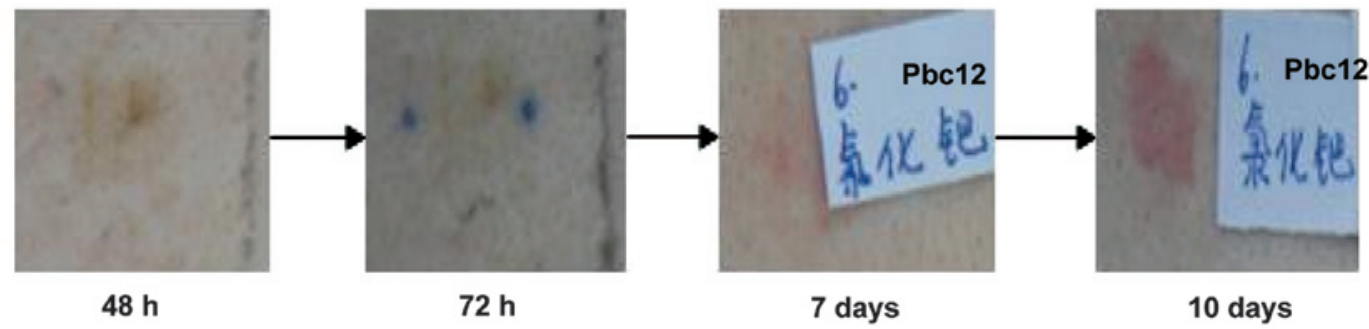

Figure 1. (A) A patch test was performed on 92 subjects with dental alloys, in order to compare the sensitivity to dental metal materials. The positive ratio of the various allergens are presented. (B) Patch test exhibited a delayed reaction. Results are usually observed at $72 \mathrm{~h}$; however, in the present study, results were observed at either $96 \mathrm{~h}, 7$ days or longer if necessary, in order to exclude false-negative results. This is an example of a delayed reaction to palladium.

were as follows: HLA-DR, forward 5'-CAGGCGAGTTTA TGTTTG-3' and reverse 5'-GATTTCCAGGTTGGCTTT-3'; GAPDH forward 5'-CCACTCCTCCACCTTTG-3' and reverse 5'-CACCACCCTGTTGCTGT-3'.

Immunoblotting. A total of $100 \mathrm{mg}$ gingival tissue sample was added to $1 \mathrm{ml}$ radioimmunoprecipitation assay (Beyotime Institute of Biotechnology, Shanghai, China) and homogenized in a homogenate apparatus at $120 \mathrm{~Hz}$ for $5 \mathrm{~min}$ prior to being centrifuged at $10,000 \mathrm{x}$ g at $4^{\circ} \mathrm{C}$ for $15 \mathrm{~min}$ in order to obtain the supernatant. Total protein $(80 \mathrm{ng})$ was extracted from gingival tissue and quantified using a Bicinchoninic Acid Protein Assay kit (Pierce Biotechnology, Inc., Rockford, USA). The cell lysates were separated by $10 \%$ sodium dodecyl sulfate-polyacrylamide gel electrophoresis (Beyotime Institute of Biotechnology), transferred to PVDF membranes (Merck Millipore Corporation, Darmstadt, Germany), and blocked with 5\% skimmed milk powder for $1 \mathrm{~h}$ at room temperature prior to being washed three times with Tris-buffered saline with $0.1 \%$ Tween 20 . The membranes were subsequently incubated with anti-HLA-DR primary antibodies (Abcam) and appropriate horseradish peroxidase-conjugated secondary antibody. The blots were visualized by chemiluminescence
(Cell Signaling Technology, Inc., Danvers, MA, USA). GAPDH (Abcam) was used as a loading control. Proteins expression levels were quantified using Image J 2x 2.1.4.7 software (National Institutes of Health, Bethesda, MA, USA).

Statistical analysis. Each experiment was performed at least three times. Data are presented as the mean \pm standard deviation. Statistical significance between groups was determined using one-way analysis of variance and a one-sample t-test. SPSS software (version 19.0; IBM SPSS, Armonk, NY, USA) was used analyze the data. $\mathrm{P}<0.05$ was considered to indicate a statistically significant difference. According to the conditions, Pearson's $\chi^{2}$ test, corrected $\chi^{2}$ test and Fisher's exact test were used.

\section{Results}

Comparative analysis of the patch test. A total of 19 metal allergens and one control were comparatively analyzed in the patch test (Fig. 1A); the control group (Vaseline ${ }^{\circledR}$ ) resulted in a negative reaction. There were 49 cases of at least one metal allergy, in which males accounted for 20 cases $(46.5 \%)$ and females accounted for 29 cases (59.2\%). There were 
A

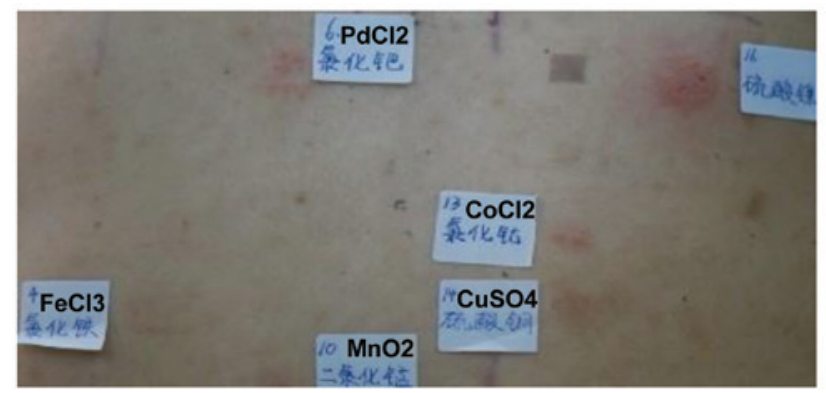

B

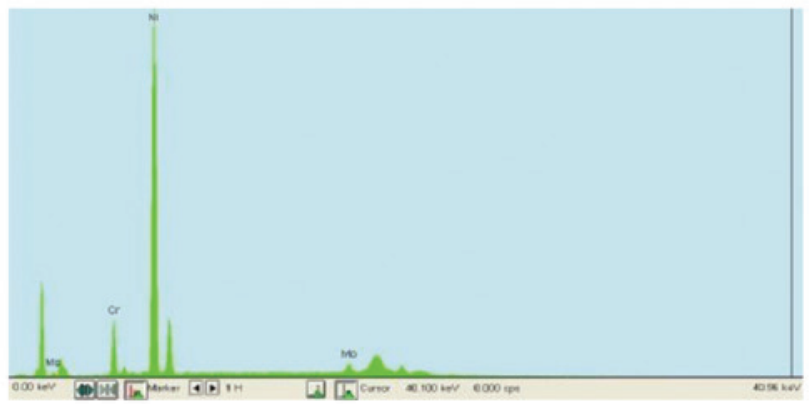

C
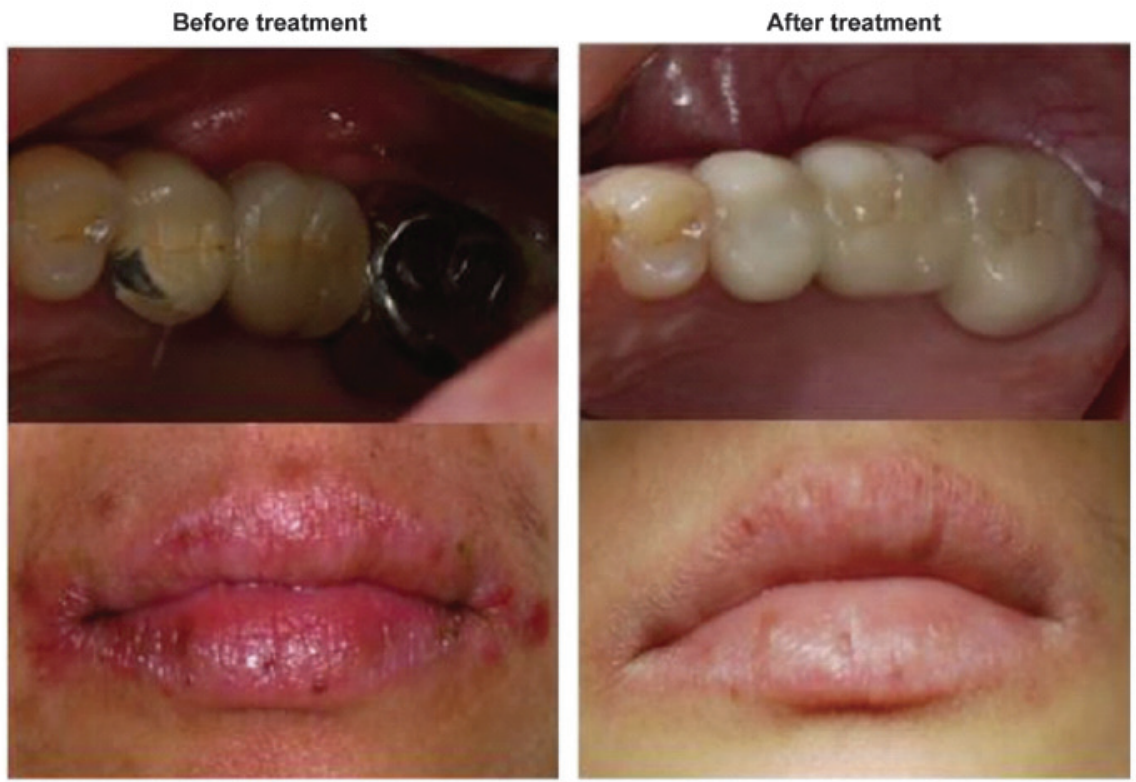

Figure 2. Case 1: Removal or replacement of a prosthesis in a patient diagnosed with metal allergy improved the majority of clinical symptoms at follow-up. Clinical features of case 1: Following restoration with a 25-27 fixed bridge, cheilitis and recurrent perioral dermatitis and eczema were observed. (A) Patch test results. Patch test results showed $\mathrm{NiSO}_{4}(++), \mathrm{PdCl}_{2}(+), \mathrm{CoCl}_{2}(+)$, and $\mathrm{MnO}_{2}(+)$.,+++ , and +++ were determined as positive reactions, subject to the allergic reaction of the patient. (B) Detection of alloy restoration composition using X-ray fluorescence microscope spectrometry (XFMS). The composition of $\mathrm{Ni}(87.52 \%), \mathrm{Cr}(9.65 \%)$ and $\mathrm{Mo}(2.65 \%)$ components detected by XFMS was in accordance with the metal component of prosthesis obtained by patch test method. (C) Before treatment: Cheilitis, recurrent skin eczema and perioral dermatitis lasted for 1 year following placement of bridge implants. After treatment: 1 month following-removal of the dental implants and replacement with a whole porcelain-fixed bridge.

positive reactions in response to at least two metal allergens in 36 patients: 12 males (27.9\%) and 24 females (49\%).

Delayed reaction. A delayed reaction was observed in the patch test. Usually, results are observed at $72 \mathrm{~h}$; however, in the present study, results were observed at $96 \mathrm{~h}, 7$ days or longer, in order to exclude false-negative results. In total, six subjects exhibited a delayed reaction, including five subjects whose first positive reaction occurred in 7 days or whose original positive reaction was more severe. Delayed reactions were observed in response to $\mathrm{Ni}, \mathrm{Hg}$ and $\mathrm{Cr}$ metal allergens. In one subject, the
Pd test sites were negative at 48 and $72 \mathrm{~h}$; however, after 7 days the Pd test sites exhibited minor erythema, and after 10 days a significant positive reaction was detected (Fig. 1B).

Patch test analysis. In patients diagnosed with metal allergy, the majority of clinical symptoms were relieved in the follow-up, following removal or replacement of the prosthesis.

Case 1. Patch test result: $\mathrm{NiSO}_{4}(++), \mathrm{PdCl}_{2}(+), \mathrm{CoCl}_{2}$ $(+), \mathrm{MnO}_{2}(+)$ (Fig. 2A). The following metal components were detected in the restoration: $\mathrm{Ni}(87.52 \%), \mathrm{Cr}(9.65 \%)$ and molybdenum (Mo; $2.65 \%$ ) by XFMS (Fig. 2B). The patient 
A

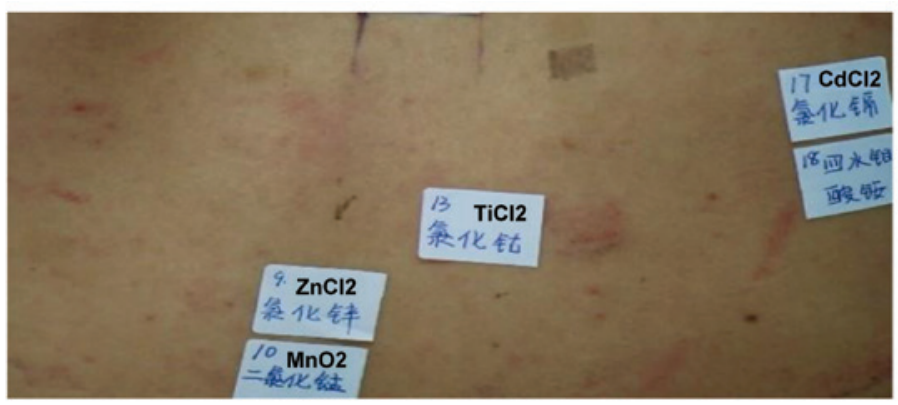

B

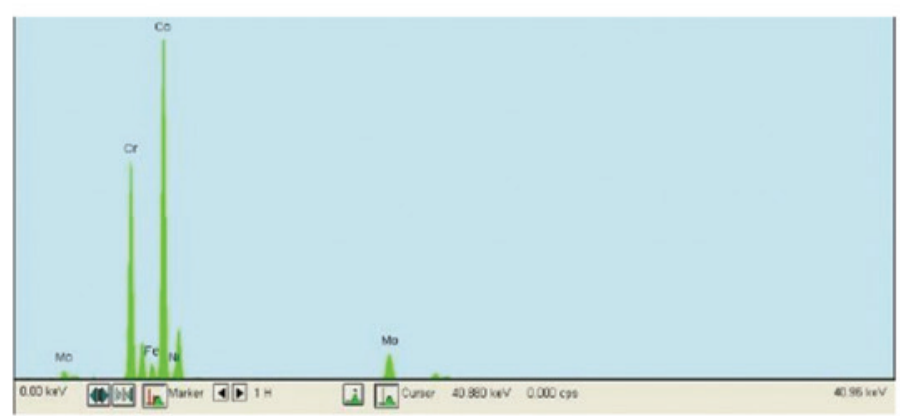

C
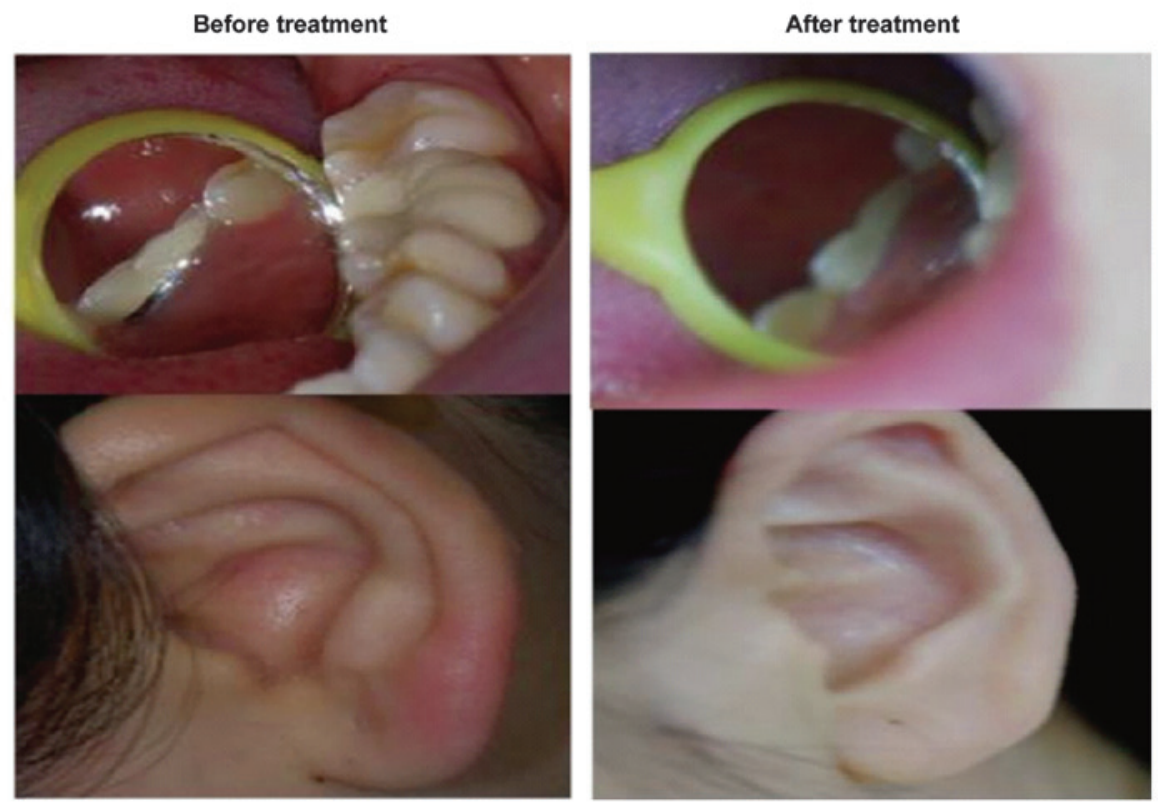

Figure 3. Clinical features of case 2. Following repair to 36 porcelain crowns, dermatitis of the face and ear were observed. (A) Patch test results. Patch test results showed $\mathrm{ZnCl}_{2}(+)$, and $\mathrm{CoCl} 2(+) .+,++$, and +++ were determined as positive reactions, subject to the allergic reaction of the patient. (B) Detection of alloy restoration composition using X-ray fluorescence microscope spectrometry (XFMS). The composition of Co (73.96\%), Cr (17.82\%) and Fe (8.22\%) components detected by XFMS was in accordance with the metal component of prosthesis obtained by patch test method. (C) Before treatment: Dermatitis of the face and ear lasted for 1 year after the patient received 36 porcelain crowns implants. After treatment: following removal of the dental implants and replacement with porcelain crowns.

had a had a strong positive reaction to $\mathrm{Ni}$ in the patch test and, using XFMS, the restoration was shown to contain up to $87.52 \% \mathrm{Ni}$; clinical symptoms occurred following dental repair, and the patch test results and metal prosthesis component test results were consistent. Therefore, removal of the metal restoration was recommended in this patient, which was replaced with a ceramic fixed bridge. The patient's symptoms were relieved after 1 month, detected on follow-up observation (Fig. 2C).

Case 2. Patch test result: $\mathrm{ZnCl}_{2}(+), \mathrm{CoCl}_{2}(+)$ (Fig. 3A). The following metal components were detected in the restoration:
Co $(73.96 \%), \mathrm{Cr}(17.82 \%)$ and iron (Fe; $8.22 \%)$ by XFMS (Fig. 3B). The patient had a strong positive reaction to Co and, using XFMS, the restoration was shown to contain up to Co 73.96\%; clinical symptoms occurred following dental repair, and the patch test results and metal prosthesis component test results were consistent. Therefore, it was determined that the clinical symptoms of patients was associated with metal prostheses sensitivity and. It was recommended that the metal restoration be removed in this patient, which was replaced with a ceramic crown. The patient's symptoms had improved at the 1 month follow-up observation (Fig. 3C). 
A

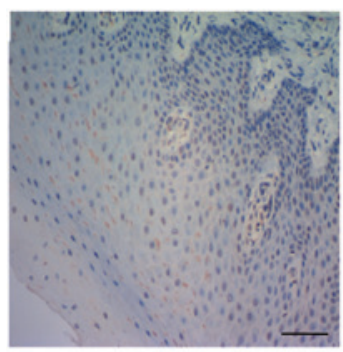

Control

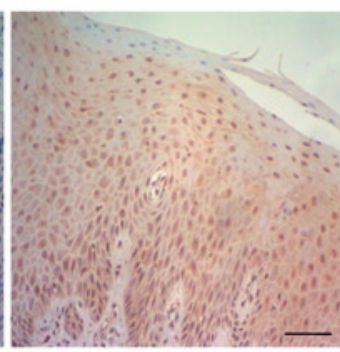

$\mathrm{Ni}-\mathrm{Cr}$ alloy

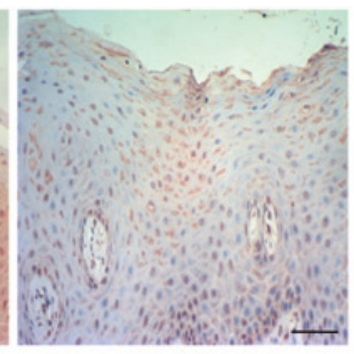

Co-Cr alloy

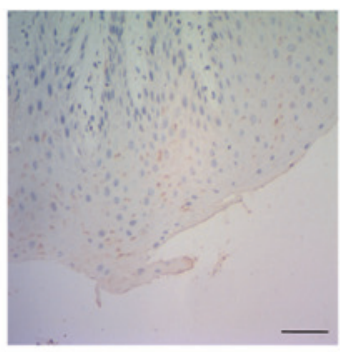

Au alloy

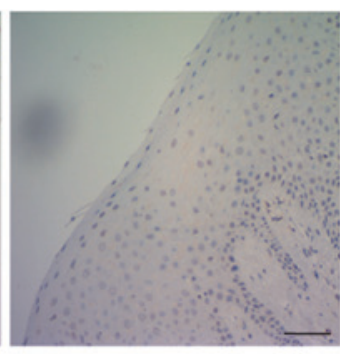

Ti alloy

B

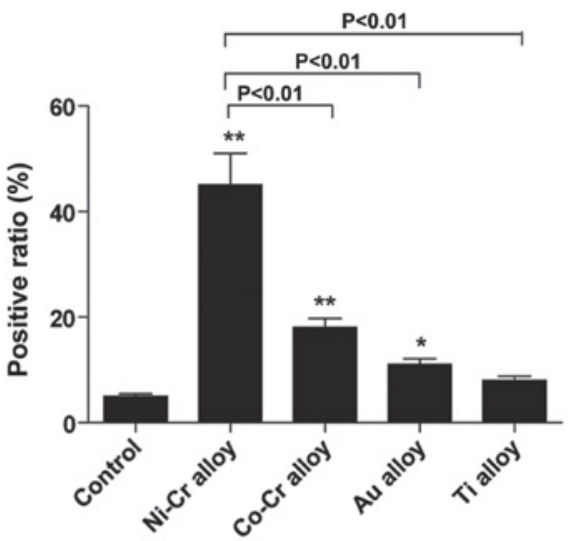

Figure 4. (A) Expression levels of human leukocyte antigen-DR in the oral mucosa epithelium of the normal gingival tissue and gingival tissue of patients with alloy restorations was determined using immunohistochemistry (horseradish peroxidase staining technique). Scale bar=0.5 $\mu \mathrm{m}$. (B) Compared with normal gingival tissue, HLA-DR positivity was increased in the alloy restoration groups. ${ }^{*} \mathrm{P}<0.05$ and ${ }^{* *} \mathrm{P}<0.01$, vs. control group. Ni, nickel; Cr, chromium; Co, cobalt; Au, gold; Ti, titanium.

A

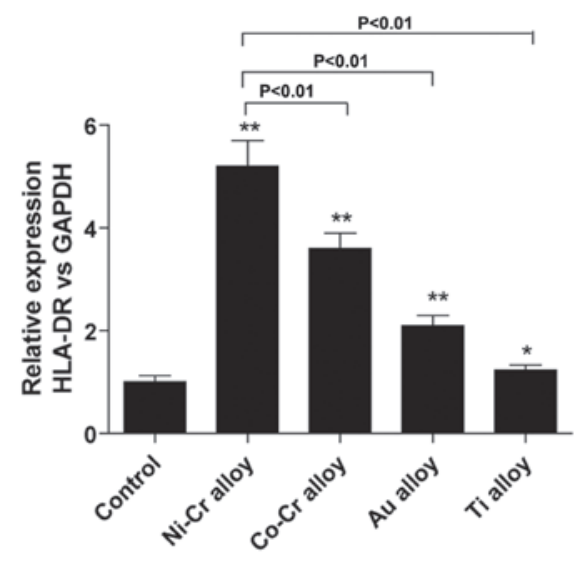

B
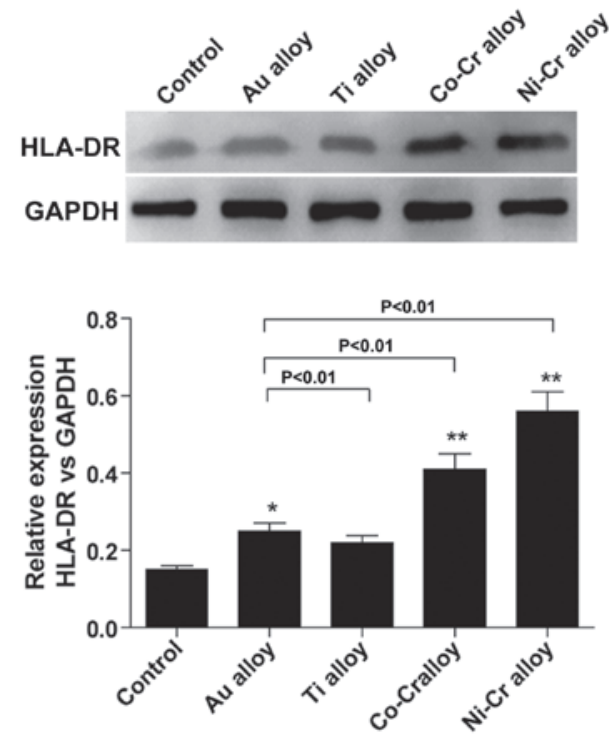

Figure 5. (A) Total RNA was extracted from gingival tissues and relative expression levels of HLA-DR were detected by polymerase chain reaction. ${ }^{*} \mathrm{P}<0.05$ and ${ }^{* *} \mathrm{P}<0.01$, vs. control. (B) Following treatment with alloy restorations for $\sim 1$ year, the protein expression levels of HLA-DR were detected by western blotting. " $\mathrm{P}<0.05$ and ${ }^{* *} \mathrm{P}<0.01$, vs. control. HLA, human leukocyte antigen; $\mathrm{Ni}$, nickel; $\mathrm{Cr}$, chromium; Co, cobalt; Au, gold; Ti, titanium; GAPDH, glyceraldehyde 3-phosphate dehydrogenase.

Expression levels of HLA-DR in gingival tissue of patients with metal restorations. The protein expression levels were of HLA-DR were significantly higher in the gingival tissues of the patients with metal restorations, compared with gingival tissues in those without, as detected using immunohistochemistry $(\mathrm{P}<0.01)$. In normal gingival tissues, HLA-DR was visible only in a small number of interstitial cells, including lymphocytes and dendritic cells. In the patients with metal 
restorations extending submucosaly, the mucosal epithelium and connective tissue had increased protein expression levels of HLA-DR (Fig. 4A and B). A small amount of light yellow staining was observed in the cytoplasm, indicating strong positive expression.

The protein and gene expression levels of HLA-DR were significantly lower in the control group, compared with those in the other groups $(\mathrm{P}<0.01)$. The protein and gene expression levels of HLA-DR were significantly higher in the $\mathrm{Ni}-\mathrm{Cr}$ prosthesis group, compared with the other groups $(\mathrm{P}<0.01)$; followed by the $\mathrm{Co}-\mathrm{Cr}$ alloy, $\mathrm{Au}$ alloy and $\mathrm{Ti}$ alloy groups, sequentially (Fig. 5A and B).

\section{Discussion}

Metal allergies have been a concern for domestic and overseas researchers for several years. $\mathrm{Ni}, \mathrm{Cr}, \mathrm{Hg}, \mathrm{Pd}$ and $\mathrm{Co}$ are commonly used components in dental metal prostheses, and readily cause allergic reactions (10). Patients who are allergic to $\mathrm{Ni}$ and $\mathrm{Cr}$ also exhibit skin allergic reactions, including eczema caused by stainless steel jewelry (4). Patch tests are considered the most reliable method for the diagnosis of delayed-hypersensitivity reactions (type IV hypersensitivity) (5). Briefly, a patch test requires the preparation of a solution or ointment containing a certain concentration of a suspected allergen, which is then applied to the skin of patients. The response to the preparation, for example eczema-like skin lesions, are used to identify specific allergens. The patch test only exposes the skin surface to the allergen, and the allergen cannot pass through the epidermis into the dermis to cause bleeding; therefore, it is considered a safe method. In the present study, the results of patch testing indicated that the number of allergies induced by Ni (83.3\%) were significantly higher, compared with the other metals. It is well-known that $\mathrm{Ni}, \mathrm{Co}$ and $\mathrm{Cr}$ can induce allergic reactions in humans (10-12), and $\mathrm{Ni}$ is considered one of the most common contact allergens. Further evidence of marked sensitization to $\mathrm{Ni}$ and Co was provided by XFMS analysis, in which $\mathrm{Ni}(87.52 \%)$ and $\mathrm{Co}(73.96 \%)$ had markedly higher sensitivity, compared with other metal ions, including Mo $(2.65 \%)$ and $\mathrm{Fe}(8.22 \%)$. Schmidt et al (13) demonstrated that $\mathrm{Ni}$ ions activate the innate immune response by stimulating Toll-like receptor 4. However, the underlying mechanism of dental metal alloy-induced activation of hypersensitivity requires further investigation.

Allergens enter the human body at different concentrations and via different routes, resulting in uncertainty in the sensitization phase duration, which may last between 3 days and several years $(14,15)$. Furthermore, allergic reactions differ among individuals, resulting in difficulties in clinical diagnosis. Nakada et al demonstrated that allergies to cobalt appeared in patients as palm or foot pustules 1 month following receipt of a dental Co-Cr alloy crown restoration. However, following removal of the gold and restoration the patients no longer exhibited clinical symptoms at follow-up (11). Further evidence of $\mathrm{Ni}-\mathrm{Cr}$ and $\mathrm{Co}-\mathrm{Cr}$-induced delayed hypersensitivity reactions was provided by the expression of HLA-DR in the present study. Previous studies have indicated that metal ions are common allergens, which sensitize T cells and induce delayed hypersensitivity reactions through its surface receptor, HLA $(16,17)$. The significant increase in the expression levels of HLA-DR in the
$\mathrm{Ni}-\mathrm{Cr}$ and $\mathrm{Co}-\mathrm{Cr}$ groups reflected the increased delayed hypersensitivity reaction. However, the expression levels of HLA-DR in the Ti alloy group showed minimal difference, compared with the healthy control, which may be due to its biocompatibility and lack of tissue sensitization (18).

In vitro experiments have demonstrated that $\mathrm{Ni}$ can cause an inflammatory reaction in epidermal cells, increase the expression levels of interleukin (IL)-1a, IL-8 and prostaglandin E2, and induce apoptosis (10). Evidence that gold leads to gum inflammation is suggestive of sensitization. The expression levels of CD4 and CD8 in the peripheral blood of patients with $\mathrm{Ni}$ allergies is relatively high; therefore, $\mathrm{Ni}$ ions may result in allergic reactions in the oral mucosa or skin (11). Allergies are usually benign; however, symptoms, including itching, can significantly lower the quality of life of patients. Therefore, identification of metal allergies and avoiding contact with specific metal allergens is the predominant therapeutic strategy. A patch test is necessary in the diagnosis of contact allergy. Dentists require an understanding of the corrosion and allergy rates of the alloys used in restorations, in order to reduce the application of highly allergic alloys. Prior to restoration, a patch test for hypersensitive patients is recommended, and the use of different metal alloys in the same patient requires caution.

In conclusion, sensitization to, and the biological safety of metals is an important topic in dental investigations. The present study exhibited clear evidence that sensitization to certain dental metals, including $\mathrm{Ni}$ and $\mathrm{Co}$, can be identified by a patch test prior to implantation, thus providing guidance for dental clinicians in the selection of repair materials.

\section{Acknowledgements}

This study was supported by the 2010 Shanghai Committee of Science and Technology, China (grant. no. 10411950900).

\section{References}

1. Lundström IM: Allergy and corrosion of dental materials in patients with oral lichen planus. Int J Oral Surg 13: 16-24, 1984.

2. Magnusson B, Bergman M, Bergman B and Söremark R: Nickel allergy and nickel-containing dental alloys. Scand J Dent Res 90: 163-167, 1982.

3. Gawkrodger DJ: Investigation of reactions to dental materials. $\mathrm{Br}$ J Dermatol 153: 479-485, 2005.

4. Yanagi T, Shimizu T, Abe R and Shimizu H: Zinc dental fillings and palmoplantar pustulosis. Lancet 366: 1050, 2005.

5. Zhu Z and Zhan DS: Mutagenicity of two Ni-Cr porcelain alloys. JMutagenicity of two Ni-Cr porcelain alloys. Journal of Clinical Rehabilitative Tissue Engineering Research 14: 2683-2685, 2008.

6. Bayindir F, Körkut $\mathrm{O}$ and Güngör H: Potentiodynamic polarization technique for corrosion testing of $\mathrm{Cr}-\mathrm{Co}$ and $\mathrm{Cr}-\mathrm{Ni}$ alloys in artificial saliva and soft drinks. J Mater Res Innov 14: 280-284, 2010.

7. Danaei SM, Safavi A, Roeinpeikar SM, Oshagh M, Iranpour S and Omidkhoda M: Ion release from orthodontic brackets in 3 mouthwashes: An in-vitro study. Am J Orthod Dentofacial Orthop 139: 730-734, 2011.

8. Uter W, Rämsch C, Aberer W, Ayala F, Balato A, Beliauskiene A, Fortina AB, Bircher A, Brasch J, Chowdhury MM, et al: The European baseline series in 10 European countries, 2005/2006 - results of the European Surveillance System on Contact Allergies (ESSCA). Contact Dermatitis 61: 31-38, 2009.

9. Zeng J, Wang J, Gao W, Mohammadreza A, Kelbauskas L, Zhang W, Johnson RH and Meldrum DR: Quantitative singlecell gene expression measurements of multiple genes in response to hypoxia treatment. Anal Bioanal Chem, 401: 3-13, 2011. 
10. Lidén $\mathrm{C}$ and Norberg K: Nickel on the Swedish market. Follow-up after implementation of the Nickel Directive. Contact Dermatitis 52: 29-35, 2005.

11. Nakada T, Iijima $M$, Nakayama $H$ and Maibach HI: Role of ear piercing in metal allergic contact dermatitis. Contact Dermatitis 36: 233-226, 228-233, 1997.

12. Merritt $\mathrm{K}$ and Rodrigo JJ: Immune response to synthetic materials. Sensitization of patients receiving orthopaedic implants. Clin Orthop Relat Res, 71-79, 1996.

13. Schmidt M, Raghavan B, Müller V, Vogl T, Fejer G, Tchaptchet S, Keck S, Kalis C, Nielsen PJ, Galanos C, et al: Crucial role for human Toll-like receptor 4 in the development of contact allergy to nickel. Nat Immunol 11: 814-819, 2010.

14. Abraham CM, Ownby DR, Peterson EL, Wegienka G, Zoratti EM, Williams LK, Joseph CL and Johnson CC: The relationship between seroatopy and symptoms of either allergic rhinitis or asthma. J Allergy Clin Immunol 119: 1099-1104, 2007.
15. Arshad SH, Tariq SM, Matthews S and Hakim E: Sensitization to common allergens and its association with allergic disorders at age 4 years: A whole population birth cohort study. Pediatrics 108: E33, 2001.

16. Thomas P, Maier S and Summer B: Allergic reactions to metal implants. Materwiss Werksttech 35: 997-1000, 2004.

17. Forte G, Petrucci F and Bocca B: Metal allergens of growing significance: Epidemiology, immunotoxicology, strategies for testing and prevention. Inflamm Allergy Drug Targets 7: 145-162, 2008.

18. Harloff T, Hönle W, Holzwarth U, Bader R, Thomas P and Schuh A: Titanium allergy or not? 'Impurity' of titanium implant materials. Health 2: 306-310, 2010. 